Article

\title{
Electrochemical Performance of a Lithium Ion Battery with Different Nanoporous Current Collectors
}

\author{
Huajun Feng, Yuan Chen and Yihua Wang *(i) \\ School of Engineering, Republic Polytechnic, Singapore 738964, Singapore; feng_huajun2@rp.edu.sg (H.F.); \\ chenyuancn411@outlook.com (Y.C.) \\ * Correspondence: wang_yihua@rp.edu.sg; Tel.: +65-6697-1058
}

Received: 26 November 2018; Accepted: 31 January 2019; Published: 12 February 2019

\begin{abstract}
In this work, we use ultrasonication and chemical etching agents to assist preparation of metal current collectors with nano-scale pores on the surface. Four different current collectors (copper foil, copper foam, aluminum foil, and aluminum foam) are prepared. The preparation parameters, ultrasonic time and etching agent concentration, are investigated and optimized accordingly. The morphologies of the as-prepared current collectors are observed under a scanning electronic microscope. Soft-packed lithium ion batteries with various current collectors are fabricated and tested. The prepared lithium ion batteries show good long-term cycle stability. The nanoporous structure of the current collector has little impact on the improvement of battery capacity under slow charging/discharging rates but has a positive impact on capacity retention under fast charging/discharging rates.
\end{abstract}

Keywords: lithium ion battery; current collector; nano porous structure; ultrasonic preparation

\section{Introduction}

Rechargeable lithium ion batteries are a key technology in the electronics market [1-3]. The basic electrochemical principle of a lithium ion battery includes a typical intercalation reaction. Lithium ions are inserted into (or extracted from) an open host-like structure with a concurrent addition (or removal) of electrons [4-6]. However, lithium ion batteries fall short of satisfying the needs for safety and fast charging/discharging performance, which are highly desirable for applications in portable devices, power tools, electric vehicles, and efficient use of renewable energies [7-9].

Ultrasonic technology has been widely applied to chemical synthesis and material processing due to its unique medium condition. Most ultrasonic effects occur in a liquid medium, where sound pressures can disrupt the continuum of the liquid phase [5,6]. Due to the high frequencies of ultrasonic waves, oscillating air bubbles are generated, which drastically change the liquid status. Extremely rapid heating or cooling, and pressures of up to several hundred mega Pascals, are observed in transient cavities, while the bulk of the liquid remains at an ambient temperature and pressure $[10,11]$. In 2010, Skorb's group reported that a metal aluminum film with nanopores on parts of its surface was obtained in water using ultrasound cell disrupting equipment [12]. Great efforts have been made to prepare a nanoporous structure on different substrates. Large scale preparation of nanopores on metal film using ultrasound waves is very difficult due to the size limitations of the ultrasonic probe and the complexity of the procedures [13-16]. 
In this paper, a low-cost ultrasonic etching method has been developed to prepare nanoporous metal current collectors, namely copper metal for the anode and aluminum metal for the cathode. A streamlined process from the material preparation to the production of a pouch cell lithium ion battery is developed accordingly. The electrochemical properties, cycle stability, and capacity retention of the prepared batteries under fast charging/discharging rates are studied.

\section{Experimental}

\subsection{Preparation of Nanoporous Metal Current Collectors}

Commercial metal current collectors from Sumitomo Electric Group (Osaka, Japan) were used as metal substrates to produce nanopores on the surface. Four different current collectors, copper foil and copper foam for the anode, and aluminum foil and aluminum foam for the cathode, were selected as the starting materials. The aluminum/copper foam has a thickness of $1 \mathrm{~mm}$ and a maximum porosity of $98 \%$. The aluminum/copper foil has a thickness of $25 \mu \mathrm{m}$. To produce nanopores on the surface, the metal current collectors were cut into small pieces and soaked in a glass container filled with the diluted etching agent solution. A chloride acid solution (37\%) and ammonia solution (25\%) from Sigma-Aldrich (St. Louis, MO, USA) were used as the original etching agents to form nanopores on the surface of aluminum and copper, respectively. The original chloride acid solution and ammonia solution were further diluted with de-ionized water to appropriate concentrations for the etching process. The container was then transferred into an ultrasonic cleaner for treatment at room temperature. The ultrasonically treated current collectors were then rinsed with de-ionized water and dried at $110{ }^{\circ} \mathrm{C}$ overnight in an oven. After drying, a scanning electron microscope (SEM) was used to observe the morphology of the as-prepared current collectors. An ultrasonic cleaner DUSA-200B with $12 \mathrm{~L}$ of capacity, 200 Watt of power, and $40 \mathrm{KHz}$ of frequency from Mex Quest (Singapore) was used as the ultrasonic source. The surface morphology of the metal current collectors after ultrasonic treatment was revealed with a JEOL JSM-6701F Scanning Electron Microscope (SEM).

\subsection{Battery Assembly and Electrochemical Test of Lithium Ion Batteries with Nanoporous Current Collectors}

After the preparation of nanopores on the surface (copper or aluminum), current collectors were used to assemble a soft-packed lithium ion battery. The whole production procedure is shown in Figure 1, which includes a series of steps beginning from the nanofoam current collector preparation, slurry coating, electrode production, and battery assembly, to the electrochemical tests.

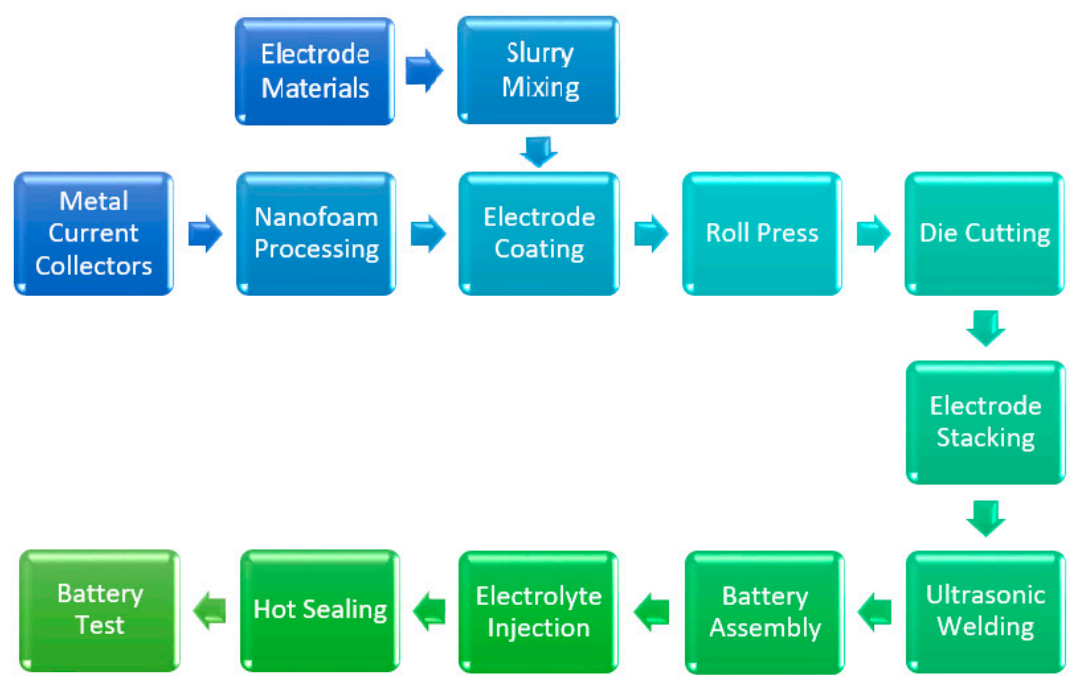

Figure 1. Fabrication process of a high-performance lithium ion battery with nano-porous current collectors. 
Nickel manganese cobalt oxide (NMC, $162 \mathrm{mAh} / \mathrm{g})$, graphite $(325 \mathrm{mAh} / \mathrm{g})$, conductive acetylene black, and poly (vinylidene fluoride) (PVDF) were purchased from MTI Corporation as electrode materials. N-Methyl-2-pyrrolidone (NMP) was purchase from Sigma Aldrich as an organic solvent to mix the electrode materials. The mixing formulations for the cathode and anode slurries are shown in Table 1. The slurries were coated on the surface of the as-prepared current collectors with a semi-automatic coating machine. A mechanical blade with an adjustable gap was used to control the coating thickness at $400 \mu \mathrm{m}$. NMC was used as the active cathode material coated on various aluminum current collectors, and graphite as the active anode material coated on various copper current collectors. Then, the coated current collector sheets were transferred to a vacuum oven and dried at $100{ }^{\circ} \mathrm{C}$ for $8 \mathrm{~h}$. After drying, the sheets went through a rolling press machine with an $80 \mu \mathrm{m}$ gap to densify the coating layer and smooth the rough surface for the next step.

Table 1. Mixing formulations of the cathode and anode slurries. NMC, Nickel manganese cobalt oxide; NMP, N-Methyl-2-pyrrolidone; PVDF, poly (vinylidene fluoride).

\begin{tabular}{ccc}
\hline Slurry & Material & Amount (g) \\
\hline \multirow{3}{*}{ Cathode Slurry } & NMC & 20 \\
& NMP solvent & 38 \\
& PVDF & 1.2 \\
& Conductive ethylene black & 1.2 \\
\hline \multirow{2}{*}{ Anode Slurry } & Graphite & 36 \\
& NMP solvent & 30 \\
& PVDF & 1.8 \\
\hline
\end{tabular}

A die cutter machine was used to cut the coated sheets into a regular rectangle shape $\left(70 \times 100 \mathrm{~mm}^{2}\right)$. After that, the cathode sheet, anode sheet, and separator film were stacked together sequentially to make the battery core. Celgard 2325 with a thickness of $25 \mu \mathrm{m}$ was used as the separator film. Then, nickel tab and aluminum tab were welded onto the outstanding part of the battery core using an ultrasonic welder (MTI Corporation, Richmond, CA, USA). Finally, the injection of liquid electrolyte (8 $\mathrm{mL}$ of $1.0 \mathrm{M} \mathrm{LiPF}_{6}$ in 1:1 EC/DEC from MTI Corporation) and the hot-sealing of the battery core were conducted in an argon-filled glovebox (VAC Controlled Atmosphere System) to complete the whole assembly of the lithium ion battery. The electrochemical performance of the prepared lithium ion batteries was tested with a Neware battery testing analyzer. During the cycling tests, the battery was charged to $3.9 \mathrm{~V}$ with a constant current and discharged to $3.0 \mathrm{~V}$ with the same constant current; we then repeated the charge/discharge process for more cycles. The whole cycling process and the setup of electrochemical parameters were programmed with the Neware controller software.

\section{Results and Discussion}

\subsection{Formation of a Nanoporous Structure on the Surface of Metal Current Collectors}

An ultrasonic etching method has been developed to produce nano-scale pores on the surfaces of different metal current collectors, which were then assembled into a lithium ion battery for electrochemical tests. For copper foil and copper foam, diluted ammonia solution was used as the etching agent to react with the copper metal in an aqueous solution in the presence of oxygen. Water-soluble $\mathrm{Cu}\left(\mathrm{NH}_{3}\right)_{4}{ }^{2+}$ complexes were produced and diffused into the surrounding aqueous solution, leaving nanoscale pores to be formed on the surface of the copper foam framework. For aluminum foil and aluminum foam, diluted chloride acid solution was used as the etching agent, and ultrasonication was used to accelerate the reaction process between the aluminum and chloride acid. 
The reaction of the copper metal with the ammonia solution is as follows:

$$
2 \mathrm{Cu}+\mathrm{O}_{2}+8 \mathrm{NH}_{3}+2 \mathrm{H}_{2} \mathrm{O} \rightarrow 4 \mathrm{OH}^{-}+2 \mathrm{Cu}\left(\mathrm{NH}_{3}\right)_{4}^{2+}
$$

The reaction of the aluminum metal with the chloride acid solution is as follows:

$$
2 \mathrm{Al}+6 \mathrm{H}^{+} \rightarrow 2 \mathrm{Al}^{3+}+3 \mathrm{H}_{2}
$$

Figure 2 shows the effects of ultrasonication and etching agents on the formation of nanoscale pores on the surface of the metal current collectors, the copper/aluminum foils. The formation of nanopores reduces the weight of the metal current collectors; this weight loss indicates the extensiveness of the nanopore formation. Before ultrasonic etching, the initial weight of the current collector sheet was recorded. After etching for a certain time (10, 30, and $60 \mathrm{~min})$, the sheet was taken out of the solution and rinsed with de-ionized water to neutral $(\mathrm{pH}=7)$. After drying on a $120^{\circ} \mathrm{C}$ hotplate for $10 \mathrm{~min}$ to remove the residue water, the sheet was weighed again. As showed in Figure 2, without ultrasonication, there was little weight loss observed regardless of chemical agents, indicating that no reaction occurred. However, after one hour of ultrasonication with certain amounts of chemical agents $(0.1 \%$ chloride acid for aluminum and $0.03 \%$ ammonia for copper), an obvious weight loss was observed. The weight loss of the copper and aluminum foils reached $3.2 \%$ and $2.7 \%$, respectively. With the increase of chemical agent concentrations, the weight loss increased, damaging the integrity of the metal foils and making them unsuitable to be used as current collectors. The copper and aluminum foams showed similar reaction results to their foil counterparts. It is concluded that the assistance of ultrasonication can accelerate the reaction process between a metal foil and a chemical agent. Therefore, in the following studies, $0.1 \%$ chloride acid and $0.03 \%$ ammonia were applied to form nanopores for aluminum and copper, respectively.

Kinetics of this heterogeneous reaction include the following five steps: (1) Diffusion of the chemical agent to the metal surface; (2) adsorption of the chemical agent; (3) interface chemical reaction at the metal solid surface with the chemical agent; (4) desorption; and (5) diffusion of the reaction products to the bulk solution [10-12]. Generally, the reaction is determined by a slow interface chemical reaction. However, the assistance of ultrasonication can create a unique oscillating environment, which may produce transient cavities at the interface with high temperature and pressure. This will greatly accelerate the interface reaction process. In addition, ultrasonic oscillation can increase the dissolution of oxygen in water and accelerate the diffusion of chemical agents to a metal surface $[17,18]$. These benefits are also helpful for the increase in reaction rate. As a result, a greater weight loss of copper was observed and more nanopores were formed within a fixed time period.

Figure 3 shows the surface morphologies of four different current collectors before and after ultrasonication treatment. The original copper foil and aluminum foil have smooth surfaces without porous structures on the surface. However, after ultrasonic treatment, many nano-sized pores were generated on the surface due to ultrasonic etching. The original copper foam and aluminum foam are full of large open pores hundreds of micrometers in size, which are observable by the naked eye. The beam of the foam framework was smooth with very few nanopores before ultrasonication. However, after ultrasonic treatment, the smooth beam surface was etched and became rough and full of nano-sized pores. 



Figure 2. Effects of ultrasonication and the etching agent on the formation of nanopores on the surfaces of (a) aluminum and (b) copper foil. 

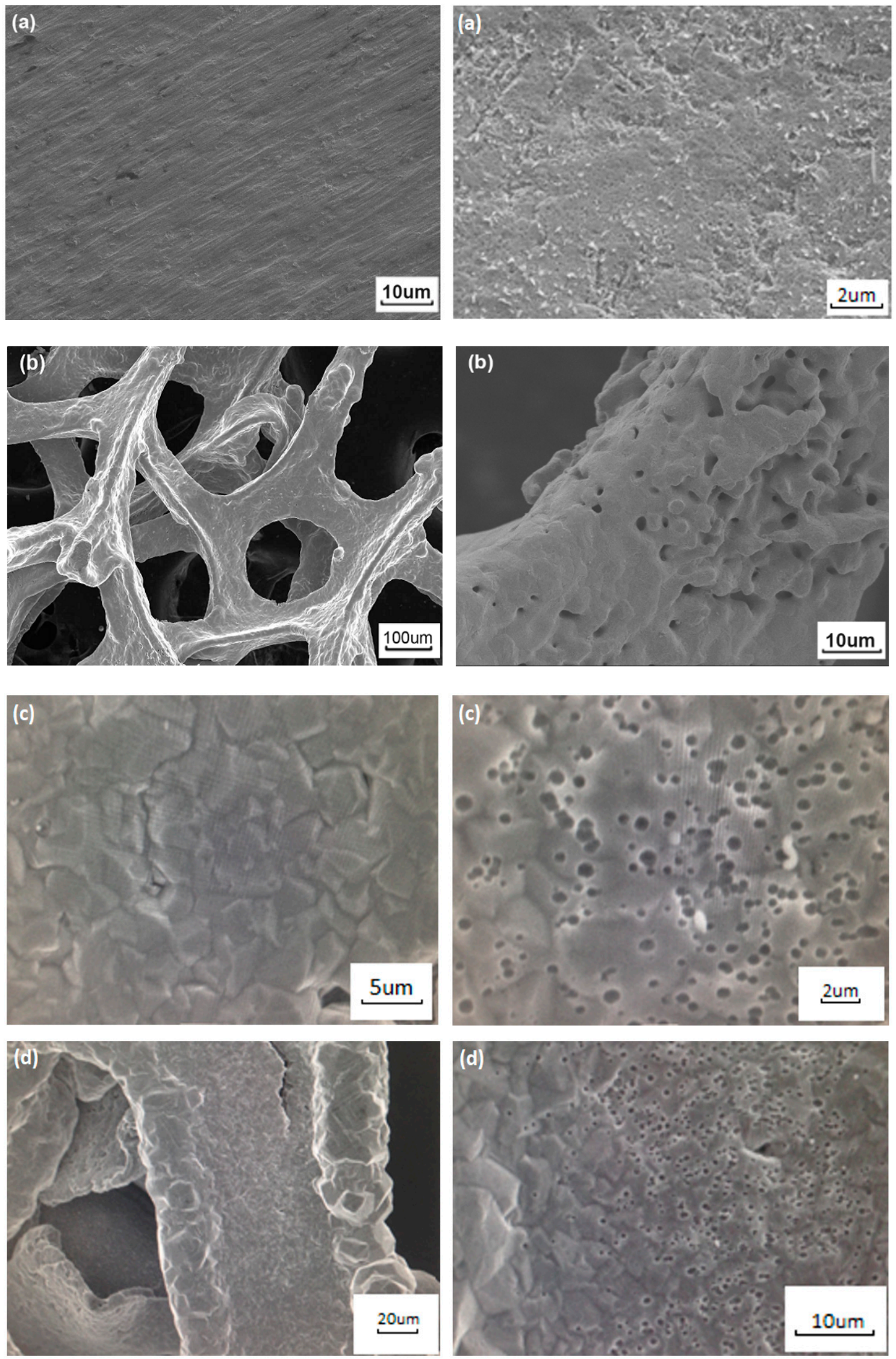

Figure 3. High resolution SEM images of the surface before (left) and after (right) ultrasonication of (a) copper foil, (b) copper foam, (c) aluminum foil, and (d) aluminum foam. 


\subsection{Electrochemical Performance of Lithium Ion Batteries with Different Nanoporous Metal Current Collectors}

Soft-packed lithium ion batteries with a volume size of $(3.5 \pm 0.1 \mathrm{~cm} \times 3.0 \pm 0.1 \mathrm{~cm} \times 0.2 \pm 0.01$ $\mathrm{cm})$ were prepared and tested with a total of 16 different current collector matches. The word "nano" means the ultrasonically treated current collector. Table 2 presents the capacity density results for 16 batteries (in weight and in volume) with different current collector matches under a $0.5 \mathrm{C}$ rate. Figure 4 visualizes the results of Table 2 for clarification. It is concluded that the batteries can be classified into three different levels based on their capacity density. The first-class batteries, with the highest capacity density of $>350 \mathrm{mAh} / \mathrm{cm}^{3}$, have a current collector match of the aluminum foam cathode and the copper foam anode, independent of nanopore formation. The second-class batteries, which have middle capacity density of between 250 and $300 \mathrm{mAh} / \mathrm{cm}^{3}$, are made of an aluminum foam cathode and copper foil anode, regardless of nanopore formation. Batteries with aluminum foil as the cathode, regardless of nanopore formation, belong to the third class, with the lowest capacity density, around $200 \mathrm{mAh} / \mathrm{cm}^{3}$. Two different electrode situations may affect the battery capacities. One is the existence of large micro-sized pores on the metal foam sheet, which can hold more active electrode materials. The other is the different capacity densities between the graphite anode (about $320 \mathrm{mAh} / \mathrm{g}$ ) and the NMC cathode (about $160 \mathrm{mAh} / \mathrm{g}$ ). This difference indicates that an increase in the amount of the NMC cathode will have a greater effect on the whole battery's capacity.

Table 2. Capacity densities of lithium ion batteries with various current collectors.

\begin{tabular}{|c|c|c|c|c|c|c|c|}
\hline Battery & $\begin{array}{c}\text { Capacity } \\
\text { (mAh) }\end{array}$ & Weight (g) & $\begin{array}{l}\text { Length } \\
(\mathrm{cm})\end{array}$ & $\begin{array}{l}\text { Width } \\
\text { (cm) }\end{array}$ & $\begin{array}{l}\text { Height } \\
\text { (cm) }\end{array}$ & $\begin{array}{l}\text { Weight Density } \\
\quad\left(\mathrm{mAh} \mathrm{g}^{-1}\right)\end{array}$ & $\begin{array}{l}\text { Volume Density } \\
\left(\mathrm{mAh} \mathrm{cm}^{-3}\right)\end{array}$ \\
\hline Al Foil-Cu Foil Nano & 413.8 & 6.57 & $3.5 \pm 0.1$ & $3.0 \pm 0.1$ & $0.2 \pm 0.01$ & 62.98 & 197.05 \\
\hline Al Foil Nano-Cu Foam & 421.35 & 8.02 & $3.5 \pm 0.1$ & $3.0 \pm 0.1$ & $0.2 \pm 0.01$ & 52.54 & 200.64 \\
\hline Al Foil Nano-Cu Foam Nano & 423.12 & 8.64 & $3.5 \pm 0.1$ & $3.0 \pm 0.1$ & $0.2 \pm 0.01$ & 48.97 & 201.49 \\
\hline Al Foil Nano-Cu Foil Nano & 423.68 & 7.43 & $3.5 \pm 0.1$ & $3.0 \pm 0.1$ & $0.2 \pm 0.01$ & 57.02 & 201.75 \\
\hline Al Foil-Cu Foil & 426.14 & 6.85 & $3.5 \pm 0.1$ & $3.0 \pm 0.1$ & $0.2 \pm 0.01$ & 62.21 & 202.92 \\
\hline Al Foil Nano-Cu Foil & 426.15 & 7.41 & $3.5 \pm 0.1$ & $3.0 \pm 0.1$ & $0.2 \pm 0.01$ & 57.51 & 202.93 \\
\hline Al Foil-Cu Foam & 435.81 & 7.92 & $3.5 \pm 0.1$ & $3.0 \pm 0.1$ & $0.2 \pm 0.01$ & 55.03 & 207.53 \\
\hline Al Foil-Cu Foam Nano & 437.28 & 8.08 & $3.5 \pm 0.1$ & $3.0 \pm 0.1$ & $0.2 \pm 0.01$ & 54.12 & 208.23 \\
\hline Al Foam-Cu Foil Nano & 603.23 & 7.39 & $3.5 \pm 0.1$ & $3.0 \pm 0.1$ & $0.2 \pm 0.01$ & 81.63 & 287.25 \\
\hline Al Foam-Cu Foil & 607.19 & 7.72 & $3.5 \pm 0.1$ & $3.0 \pm 0.1$ & $0.2 \pm 0.01$ & 78.65 & 289.14 \\
\hline Al Foam Nano-Cu Foil Nano & 614.47 & 7.57 & $3.5 \pm 0.1$ & $3.0 \pm 0.1$ & $0.2 \pm 0.01$ & 81.17 & 292.61 \\
\hline Al Foam Nano-Cu Foil & 629.9 & 7.95 & $3.5 \pm 0.1$ & $3.0 \pm 0.1$ & $0.2 \pm 0.01$ & 79.23 & 299.95 \\
\hline Al Foam-Cu Foam & 787.97 & 7.86 & $3.5 \pm 0.1$ & $3.0 \pm 0.1$ & $0.2 \pm 0.01$ & 100.25 & 375.22 \\
\hline Al Foam Nano-Cu Foam & 797.4 & 8.16 & $3.5 \pm 0.1$ & $3.0 \pm 0.1$ & $0.2 \pm 0.01$ & 97.72 & 379.71 \\
\hline Al Foam-Cu Foam Nano & 799.78 & 8.33 & $3.5 \pm 0.1$ & $3.0 \pm 0.1$ & $0.2 \pm 0.01$ & 96.01 & 380.85 \\
\hline Al Foam Nano-Cu Foam Nano & 808.57 & 8.31 & $3.5 \pm 0.1$ & $3.0 \pm 0.1$ & $0.2 \pm 0.01$ & 97.3 & 385.03 \\
\hline
\end{tabular}

Note: Al—Aluminum; $\mathrm{Cu}-$ Copper.

Figure 5 shows the electrochemical performance of lithium ion batteries under different rates, continuously changing from $0.1 \mathrm{C}, 0.2 \mathrm{C}, 0.5 \mathrm{C}, 1 \mathrm{C}, 2 \mathrm{C}$ to $5 \mathrm{C}$, and then back to $0.1 \mathrm{C}$. The $\mathrm{nC}$ rate is defined as the charge/discharge of the battery with a constant current in $1 / \mathrm{n}$ hours. For $1 \mathrm{C}$, the $\mathrm{n}$ is equal to 1 ; for $0.2 \mathrm{C}$, the $\mathrm{n}$ is $5 \mathrm{~h}$, and so on for other rates. The detailed cycling experimental procedure and parameters are described in Section 2.2. With the increase of current, the capacity of the lithium ion battery decreases slowly. Under low currents, the capacities of all batteries are classified into three obvious levels. The nanoporous structure of the current collectors has no obvious effects on battery capacity. However, with an increase of currents, the capacity difference among the three battery classes becomes narrower. Table 3 shows the average capacities and percentage improvements due to a nanoporous structure under a $5 \mathrm{C}$ rate for different current collector matches. The results indicate that, in most cases, batteries with a nanoporous structure keep higher capacity retention at high currents than common batteries without nanopores. In theory, a porous structure can provide sufficient inner free space to absorb large volume expansions and improve cycling stability. The formation of nanopores on the surface of the metal current collector is beneficial to capacity stability. In summary, different current collectors are one of the major causes resulting in different battery capacities. Currently, we are doing more experiments and analyses to deeply investigate the changes of battery composition and micro-structures. We are trying to understand the relationship of 
these changes to different current collectors and their comprehensive effects on the improvement of battery capacity.

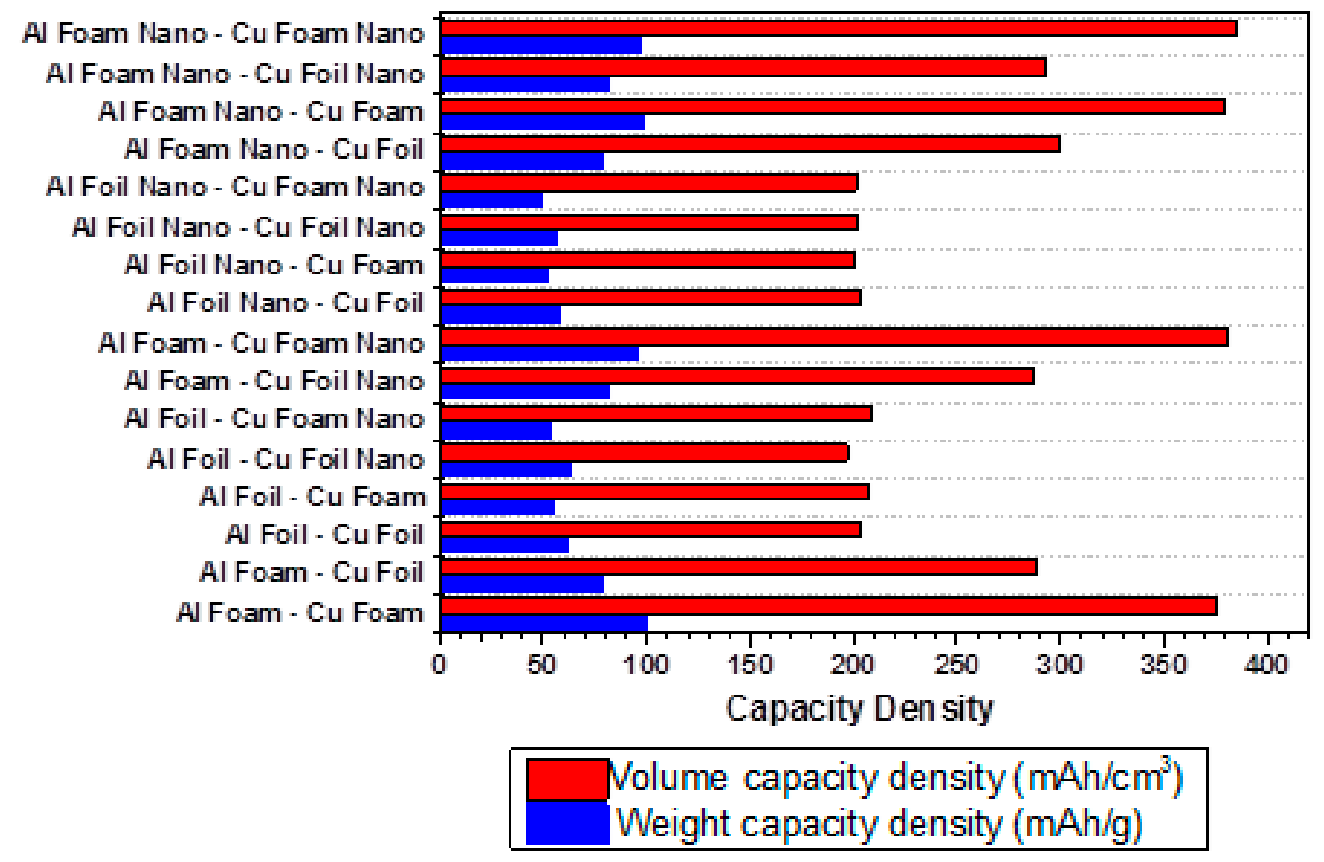

Figure 4. Volume capacity comparison of lithium ion batteries with various metal current collectors.



Figure 5. Cycling stability of lithium ion batteries with various metal current collectors.

Figure 6 shows the cycling stability of the prepared lithium ion battery with aluminum and copper nanofoam current collectors under a $1 \mathrm{C}$ rate. For comparison, two soft-packed commercial lithium ion batteries, with nominal capacities of $6000 \mathrm{mAh}$ each, were purchased from Sanyo Electronics and LG Batteries. The two commercial batteries were tested under the same electrochemical conditions as the prepared batteries. After 500 cycles, the capacity dropdown of the prepared battery $(10.1 \%)$ was obviously better than that of the commercial Sanyo and LG batteries (19.9\% and 22.5\%, respectively). 
Table 3. Average capacity of lithium ion batteries with different current collector matches under a $5 \mathrm{C}$ rate.

\begin{tabular}{ccc}
\hline Current Collector Match & $\begin{array}{c}\text { Average Capacity }\left(\times \mathbf{1 0}^{-\mathbf{1}} \text { Ah) }\right. \\
\text { under 5C Rate }\end{array}$ & $\begin{array}{c}\text { Percent of Change with } \\
\text { Nanoporous Structure }\end{array}$ \\
\hline Al Foam-Cu Foam & 1.899537 & - \\
Al Foam Nano-Cu Foam & 1.830201 & $-3.65 \%$ \\
Al Foam-Cu Foam Nano & 1.958342 & $3.10 \%$ \\
Al Foam Nano-Cu Foam Nano & 2.124354 & $11.84 \%$ \\
Al Foam-Cu Foil & 1.244933 & - \\
Al Foam-Cu Foil Nano & 1.387696 & $11.47 \%$ \\
Al Foam Nano-Cu Foil & 1.322247 & $6.21 \%$ \\
Al Foam Nano-Cu Foil Nano & 1.512555 & $21.50 \%$ \\
Al Foil-Cu Foil & 0.713199 & - \\
Al Foil Nano-Cu Foil & 0.788985 & $10.63 \%$ \\
Al Foil-Cu Foil Nano & 0.921765 & $29.24 \%$ \\
Al Foil Nano-Cu Foil Nano & 0.878092 & $23.12 \%$ \\
Al Foil-Cu Foam & 1.046454 & - \\
Al Foil-Cu Foam Nano & 1.292135 & $23.48 \%$ \\
Al Foil Nano-Cu Foam & 1.026151 & $-1.94 \%$ \\
Al Foil Nano-Cu Foam Nano & 1.031377 & $-1.44 \%$ \\
\hline
\end{tabular}



Figure 6. Long-term cycling performance of a self-prepared lithium ion battery in comparison to two commercial batteries.

\section{Conclusions}

In summary, we have successfully prepared different metal current collectors with nanoporous structures by using a combination of ultrasonication and chemical etching. The processes and parameters to prepare nanoporous metal current collectors were carefully investigated and optimized. The increase of ultrasonic time and concentration of the chemical etching agent accelerated the formation of nanoporous structures. Scanning electronic microscopy revealed nanopores on the treated surface of the metal current collectors. The as-prepared current collectors were assembled in lithium ion batteries with graphite as the active anode material and $\mathrm{LiCoMnO}_{2}$ as the active cathode material. The testing results of the assembled pouch cell batteries with nanoporous current collectors showed significant improvements in both electrode capacity and cycling stability under high charge/discharge rates. 
Author Contributions: H.F. carried out all the measurements and subsequent data analyses and prepared the manuscript. Y.C. assisted in the editing of the manuscript for submission. Y.W. helped in obtaining the funding to support this project and assisted in the data analyses, preparation, and editing of the manuscript for submission.

Funding: This research was funded by the Ministry of Education of Singapore (Grant number MOE2015-TIF-1-G-013).

Acknowledgments: The authors would like to extend their gratitude to GENPLUS Pte Ltd. for their assistance in the project by providing technical expertise and support, including evaluating the prototype and giving feedback for further enhancing of the prototype.

Conflicts of Interest: The authors declare no conflict of interest.

\section{References}

1. Tarascon, J.; Armand, M. Issues and challenges facing rechargeable lithium batteries. Nature 2001, 414, 359-367. [CrossRef] [PubMed]

2. Soloveichik, G. Battery technologies for large-scale stationary energy storage. Annu. Rev. Chem. Biomol. Eng. 2011, 2, 503-527. [CrossRef] [PubMed]

3. Xiao, J.; Yang, S.; Wan, L.; Xiao, F.; Wang, S. Electrodeposition of manganese oxide nanosheets on a continuous three dimensional nickel porous scaffold for high performance electrochemical capacitors. J. Power Sources 2014, 245, 1027-1034. [CrossRef]

4. Deng, D. Li-ion batteries: Basics, progress, and challenges. Energy Sci. Eng. 2015, 3, 385-418. [CrossRef]

5. Nitta, N.; Wu, F.; Lee, J.; Yushin, G. Li-ion battery materials: Present and future. Mater. Today 2015, 18, 252-264. [CrossRef]

6. Shen, L.; Uchaker, E.; Zhang, X.; Cao, G. Hydrogenated $\mathrm{Li}_{4} \mathrm{Ti}_{5} \mathrm{O}_{12}$ nanowire arrays for high rate lithium ion batteries. Adv. Mater. 2012, 24, 6502-6506. [CrossRef] [PubMed]

7. Li, Y.; Ou, C.; Huang, Y.; Shen, Y.; Li, N.; Zhang, H. Towards fast and ultralong-life Li-ion battery anodes: Embedding ultradispersed $\mathrm{TiO}_{2}$ quantum dots into three-dimensional porous graphene-like networks. Electrochim. Acta 2017, 246, 1183-1192. [CrossRef]

8. Prada, E.; Domenico, D.; Creff, Y.; Bernard, J.; Sauvant-Moynot, V.; Huet, F. Simplified electrochemical and thermal model of LiFePO4-graphite li-ion batteries for fast charge applications. J. Electrochem. Soc. 2012, 159, A1508-A1519. [CrossRef]

9. Martens, J.; Jammaer, J.; Bajpe, S.; Aerts, A.; Lorgouilloux, Y.; Kirschhock, C. Simple synthesis recipes of porous materials. Microporous Mesoporous Mater. 2011, 140, 2-8. [CrossRef]

10. Yoshizawa, S.; Akagi, R.; Umemura, S. Enhancement of high-intensity focused ultrasound heating by short-pulse generated cavitation. Appl. Sci. 2017, 7, 7030288. [CrossRef]

11. Skorb, E.; Fix, D.; Shchukin, D.; Möhwald, H.; Sviridov, D.; Mousa, R.; Wanderka, N.; Schäferhans, J.; Pazos-Pérez, N.; Fery, A.; et al. Sonochemical formation of metal sponges. Nanoscale 2011, 3, 985-993. [CrossRef] [PubMed]

12. Skorb, E.; Shchukin, D.G.; Möhwald, D.H.; Andreeva, D. Ultrasound-driven design of metal surface nanofoams. Nanoscale 2010, 2, 722-727. [CrossRef] [PubMed]

13. Zhang, H.; Yu, X.; Braun, P. Three-dimensional bicontinuous ultrafast-charge and -discharge bulk battery electrodes. Nat. Nanotechnol. 2011, 6, 277-281. [CrossRef] [PubMed]

14. Sakamoto, J.; Dunn, B. Hierarchical battery electrodes based on inverted opal structures. J. Mater. Chem. 2002, 12, 2859-2861. [CrossRef]

15. Pikul, J.; Zhang, H.; Cho, J.; Braun, P.; King, W. High-power lithium ion microbatteries from interdigitated three-dimensional bicontinuous nanoporous electrodes. Nat. Commun. 2013, 4, 1732-1736. [CrossRef] [PubMed]

16. Vukovic, I.; Brinke, G.; Loos, K. Block copolymer template-directed synthesis of well-ordered metallic nanostructures. Polymer 2013, 54, 2591-2605. [CrossRef] 
17. Pray, H.; Schweickert, C.; Minnich, B. Solubility of hydrogen, oxygen, nitrogen, and helium in water at elevated temperatures. Ind. Eng. Chem. 1952, 44, 1146-1151. [CrossRef]

18. Carpenter, J. New measurements of oxygen solubility in pure and natural water. Limnol. Oceanogr. 1966, 11, 264-277. [CrossRef] 